\title{
Emitting Radio Waves with Polarization Currents
}

\section{An unconventional antenna technology can focus the radio waves emitted from the acceleration of polarization currents, aiding use of the waves in communication applications.}

\author{
By Erika K. Carlson
} n conventional radio technology, radio waves are generated by accelerating charged particles, such as electrons. Now, John Singleton of Los Alamos National Laboratory, New Mexico, and colleagues have demonstrated a way to focus information carried by radio waves that are instead emitted by accelerated polarization currents-the propagation of fluctuations in the dipole moments of molecules [1]. Unlike electrons or ions, polarization currents can propagate faster than the speed of light. This factor, and the ability of these currents to radiate from a large volume, gives the technology capabilities inaccessible to conventional antennas, the researchers say.

The antenna consists of a dielectric layer sandwiched by two rows of electrodes. The researchers increase and decrease the voltage differences across pairs of opposing electrodes to manipulate dipole moments in the dielectric. Making those dipole moments larger and smaller creates a polarization

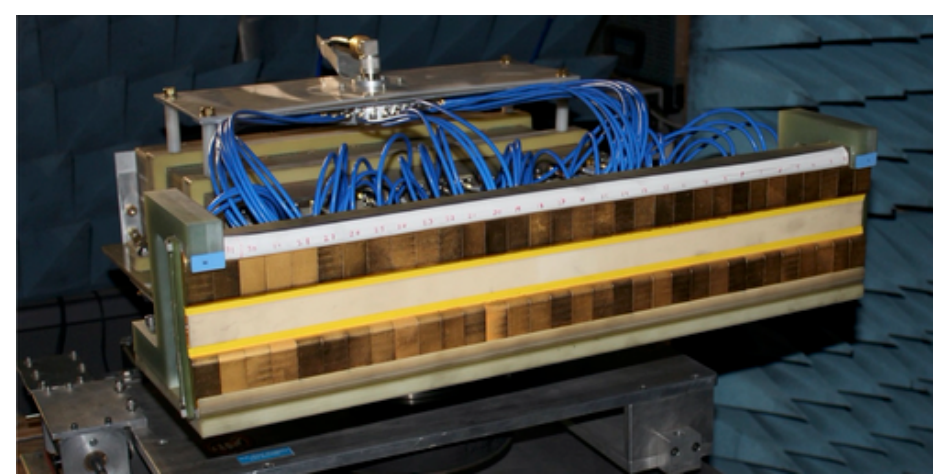

Credit: J. Singleton et al. [1] current that runs through the whole dielectric in the same way that sports fans standing and waving their arms in succession create a stadium wave. By carefully timing the voltage changes, the researchers generate a polarization current that accelerates to a speed much faster than that of light.

The current's properties grant the antenna special features. The antenna can create intense concentrations of emitted radiation that amplify a signal, similar to the way a jet accelerating through the speed of sound can create a sonic boom. That means that operators could broadcast signals in such a way that only a detector at a specific point in space can receive the signal in the same form that it was emitted, enabling a polarization-current antenna to send private wireless signals.

Erika K. Carlson is a Corresponding Editor for Physics based in New York City.

\section{REFERENCES}

1. J. Singleton et al., "Information carried by electromagnetic radiation launched from accelerated polarization currents," Phys. Rev. Applied 14, 064046 (2020). 